\title{
Characterizing the Eccentricities of Transiting Extrasolar Planets with Kepler and CoRoT
}

\author{
Eric B. Ford and Knicole D. Colón \\ University of Florida, \\ 211 Bryant Space Sciences Building, Gainesville, FL 32605, USA \\ email: eford@astro.ufl.edu
}

\begin{abstract}
Radial velocity planet searches have revealed that many giant planets have large eccentricities, in striking contrast with the giant planets in the solar system and prior theories of planet formation. The realization that many giant planets have large eccentricities raises a fundamental question: Do terrestrial-size planets of other stars typically have significantly eccentric orbits or nearly circular orbits like the Earth? While space-based missions such as CoRoT and Kepler will be capable of detecting nearly Earth-sized planets, it will be extremely challenging to measure their eccentricities using radial velocity observations. We review several ways that photometric measurements of transit light curves can constrain the eccentricity of transiting planets. In particular, photometric observations of transit durations can be used to characterize the distribution of orbital eccentricities for various populations of transiting planets (e.g., nearly Earth-sized planets in the habitable zone) without relying on radial velocity measurements. Applying this technique to rocky planets to be found by CoRoT and Kepler will enable constraints on theories for the excitation of eccentricities and tidal dissipation. We also remind observers that several short-period transiting planets are known to have significant eccentricities and caution that assuming they are on a circular orbit can reduce the probability of detecting transits, impact planning for follow-up observations, and adversely affect measurements of the physical parameters of the star and planet.
\end{abstract}

\section{Importance of Eccentric Planets}

Radial velocity surveys have detected over 200 extrasolar planets and demonstrated that many giant planets have significant orbital eccentricities. However, most extrasolar giant planets with short orbital periods -and the first planets detected in transit- have small eccentricities (Butler et al. 2006), often presumed due to tidal circularization. Thus, it is common for astronomers to assume a circular orbit when sifting through transiting planet candidates and when analyzing transit light curves to derive the physical properties of the planet and host star. However, recent discoveries have made it clear that transiting planets can have significant or even large eccentricities. In particular, the transiting planets WASP-14b (Joshi et al. 2008), GJ 436b (Maness et al. 2007; Deming et al. 2007), WASP-10b (Christian et al. 2008), TrES-1b, XO-3b (Johns-Krull et al. 2008), HD 147506b (a.k.a. HAT-P-2; Bakos et al. 2007), and HD 17156b (Gillon et al. 2007) have orbital eccentricities ranging from $\sim 0.06-0.67$. As space-based transit searches begin to detect transiting planets with greater orbital periods, even larger eccentricities will become possible, as demonstrated by the recent detection of a secondary eclipse of the radial velocity planet HD 80606b. The possibility of eccentric transiting planets presents several challenges for transit search and follow-up observations. Fortunately, transit observations also provide an opportunity to characterize the eccentricities of transiting planets, so as to constrain theories of planet formation, eccentricity excitation, and tidal effects. 


\section{Recognizing Eccentric Planets}

\section{Radial velocity and/or astrometry}

So far, the vast majority of extrasolar planets have been discovered or confirmed by radial velocity observations. Since the position and velocity of the star as a function of time depend on orbital eccentricity, both radial velocity or future astrometric observations can be used to measure orbital eccentricities, provided a sufficient number, precision, and time span of radial velocity and/or astrometric measurements. For small eccentricities, it is advantageous to describe the orbit in terms of $e \cos \omega$ and $e \sin \omega$, rather than $e$ and $\omega$, where $e$ is the eccentricity and $\omega$ is the argument of pericenter measured from the plane of the sky (Ford 2006; Shen \& Turner 2008). For eccentricities near the detection limit, radial velocities typically provide a stronger constraint on $e \cos \omega$ than $e \sin \omega$ (Laughlin et al. 2005). Below, we describe additional techniques for measuring eccentricities that can be complementary in the sense that they are more sensitive to $e \sin \omega$.

While radial velocities have proved extraordinarily valuable for probing the properties of giant planets and hot Neptunes, several considerations will make it increasingly important to consider alternative methods of measuring orbital eccentricities. First, an increasing number and fraction of exoplanets are being discovered by transit surveys that often target stars for which measuring precise radial velocities is more expensive (faint stars) or impractical (early type stars with few and/or broad spectral lines). Second, the sheer number of transiting planets expected from missions such as CoRoT and Kepler will make it challenging to obtain a sufficient number of high precision radial velocities for all exoplanets. Finally, as transit searches push towards Earth-like planets, the amplitude of the radial velocity perturbations will push the limits of detectability. If the radial velocity amplitude for a planet on a circular orbit is $K$, then for a small eccentricity the radial velocity curve will be similar to that of a similar planet on a circular orbit, but include a harmonic at half the period with an amplitude of $\sim O(e \times K)$. Even if well-timed observations are able to measure the radial velocity amplitude to confirm a candidate terrestrial planet, measuring the shape of the radial velocity curve and hence the eccentricity will be considerably more challenging.

\section{Transit Duration}

The transit duration for an eccentric transiting planet can be significantly longer or shorter than the duration for a similar planet on a circular orbit. The duration of a transit is set by the size of the star and planet, the velocity of the planet relative to the star projected onto the plane of the sky, and the viewing geometry (parameterized by the impact parameter). For a planet on a circular orbit, the velocity is set by the system mass, semi-major axis, and Kepler's law. For a planet on an eccentric orbit, the transit duration can be smaller for transits that occur near pericenter or longer for transits that occur near apocenter. The ratio of the transit duration relative to that of similar transit by a planet on a circular orbit is $(1+e \sin \omega) / \sqrt{1-e^{2}}$ (Barnes 2007; Burke 2008; Ford et al. 2008).

In order to measure orbital eccentricity from the light curve of an individual planet, fast-cadence high-precision observations are required, so as to simultaneously measure the transit duration and impact parameter. Analytic estimates of the precision suggest that the transit duration and impact parameter could be measured with considerable precision (Seager \& Mallén-Ornelas 2003; Ford et al. 2008; Carter et al. 2008). In practice, this can be complicated due to uncertainties in the limb darkening parameters, particularly for optical wavelengths. In Figure 1, we show the fractional precision for the planet's 


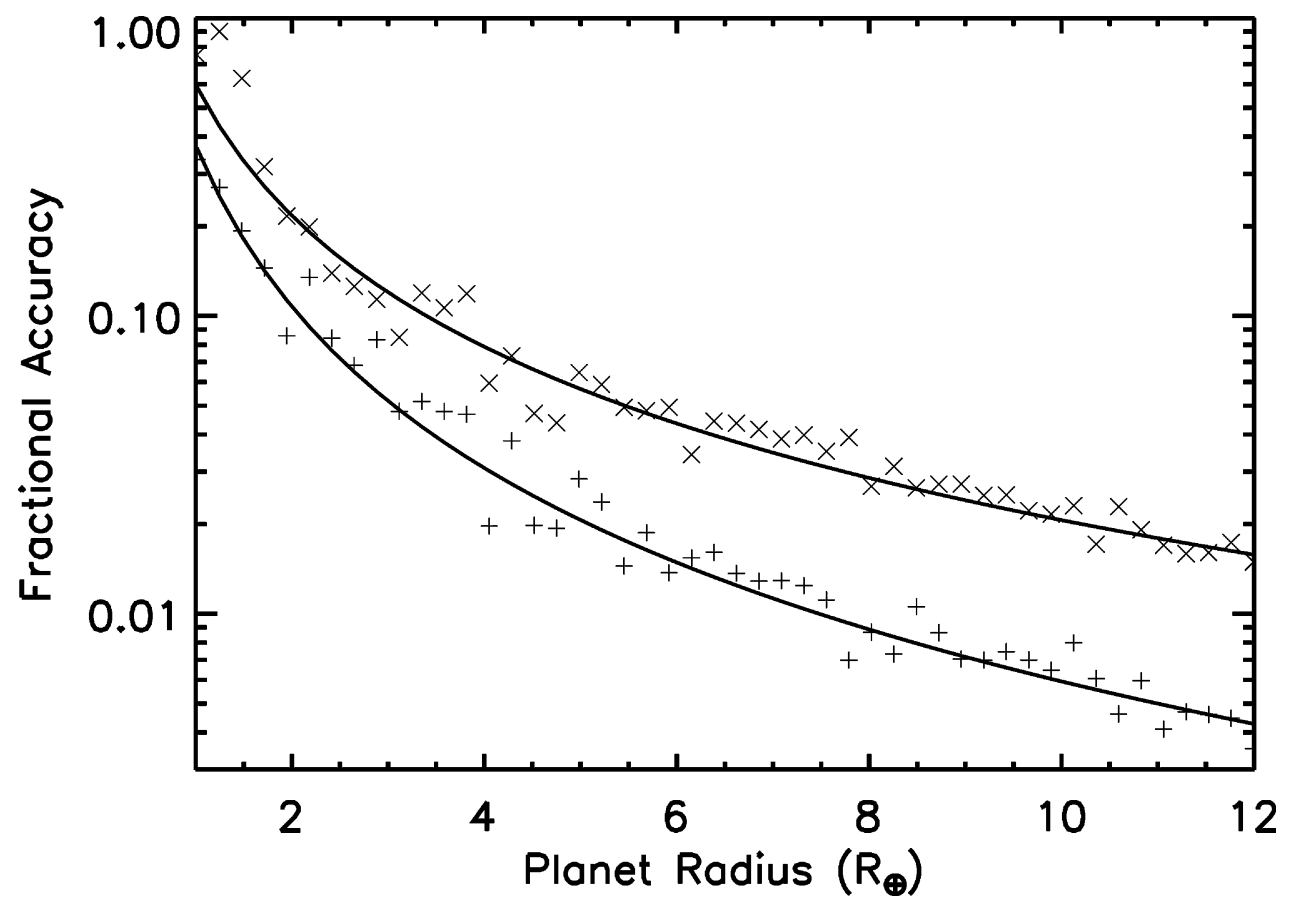

Figure 1. Fractional precision of planet velocity (projected onto the plane of the sky) using simulated Kepler observations as a function of planet radius. Here we assume that the stellar density is well determined by separate observations, so the fractional precision of the planet velocity gives the uncertainty in the measurement of $e \sin \omega$ for low eccentricities. We consider a nearly central transit of a planet orbiting a $1 M_{\oplus}$ star in a nearly circular orbit $1 \mathrm{AU}$ from the host star. The points indicate the estimated fractional accuracy of the transit duration based on fitting to simulated light curves. The pluses assume a photometric precision of $4 \times 10^{-4}$ in the $\mathrm{V}$ bandpass for each 1 minute integration, as expected for Kepler observations of a $V=12$ star. The crosses assume a photometric precision of $1.7 \times 10^{-3}$ in $z$ band for each 1 minute integration, based on the demonstrated precision of the Transit Light Curve project (Winn et al. 2007). For both cases we assume uncorrelated Gaussian noise. We simulate observations of a single transit using limb darkening parameters for the $\mathrm{V}$ band (Kepler) and $z$ band (ground) as computed by Claret (2000), but assume that both the quadratic limb darkening parameters (Mandel \& Agol 2002) must be estimated from transit observations alone (Southworth 2008). The solid curves show empirical power-law fits to our simulations for the fractional precision of transit duration. The top curve (ground) is $\simeq 0.60 \times\left(R_{\mathrm{planet}} / R_{\oplus}\right)^{-1.46}$, and the bottom curve (Kepler) is $\simeq 0.38 \times\left(R_{\text {planet }} / R_{\oplus}\right)^{-1.80}$.

velocity, as estimated from analyzing simulated light curves representative of Kepler and ground-based observations of a single transit. We simultaneously fit for the mid-time of transit, planet velocity (projected onto the plane of the sky), planet-star radius ratio, impact parameter, and the two quadratic limb darkening parameters. Assuming the star's properties are well-determined, the fractional precision of the planet velocity gives the uncertainty in the measurement of $e \sin \omega$ for low eccentricities. Even in cases where it is not practical to measure the eccentricity for individual planets, it is possible to constrain the eccentricity distribution for a given population of transiting planets (Ford et al. 2008).

When all of the above parameters are well determined, the measurement uncertainty for eccentricity will be set by the uncertainty in the mean density of the host star. At present, the stellar density can be determined by combining the stellar magnitude, parallax, spectroscopic observations of the effective temperature and metallicity, and 
stellar modeling. For stars observed by CoRoT and/or Kepler, astroseismic analyses may provide even better constraints on the stellar density.

\section{Asymmetric ingress/egress duration}

There is a slight difference in the duration of transit of ingress and egress. This effect becomes impractically small for long orbital periods, but the difference in durations of ingress and egress can reach minutes for a transiting hot-Jupiter. Nevertheless, this effect is unlikely to be useful except for constraining highly eccentric orbits.

\section{Changes in transit duration (if precessing)}

For an eccentric planet, the transit duration depends upon the orientation of the orbit relative to the line of sight. If the periapse direction is precessing (or regressing), then the transit duration will change with time. For short-period planets, general relativity and oblateness of the host star both induce precession. In multiple planet systems, the gravitational perturbations of other planets typically lead to regression of the periapse. In strongly interacting systems (short orbital periods and massive planets), this could lead to an observed modulation of the transit duration. However, in most planetary systems the perturbations will induce a secular advance of the periapse causing the change in the transit duration to accumulate with time. By measuring the transit duration over decades, one can search for a drift and place constraints on the quadrupole moment of the star and/or the presence of additional companions (Miralda-Escudé 2002; Jordan \& Bakos 2008). Even if the exact cause of the periapse advance cannot be isolated, a change in the transit duration implies a non-zero eccentricity for the vast majority of cases. The exception is for nearly grazing transits for which a small change in the inclination and hence impact parameter could have a significant effect on the transit duration (Ribas et al. 2008).

\section{Offset between primary and secondary transit}

For a planet on a circular orbit, the secondary transit occurs at the midpoint between two successive transits. For a planet on an eccentric orbit, the time of secondary transit shifts. This effect has been observed in Spitzer observations of secondary transit. This method is complementary to radial velocities, as it provides an upper limit on $e \sin \omega$ (Charbonneau et al. 2005). As a result, comparing the observed time of primary and secondary transit can improve the upper limit on the eccentricity, even for systems with extensive high-quality radial velocity observations (e.g., HD 209458; Laughlin 2005; Ford 2006). Conversely, the sensitivity of the time of secondary transit to a non-zero eccentricity implies that observations searching for a secondary transit must be mindful of a possible eccentricity. Even when there is no indication of an eccentricity from early radial velocity observations, observations searching for a secondary transit need to span a time interval set by the uncertainty in $e \sin \omega$.

\section{Differing durations of primary and secondary transit}

A non-zero eccentricity affects the duration of both primary and secondary transit, causing the durations to differ. For small eccentricities, the fractional magnitude of the difference is $\sim O(e \cos \omega)$, making it complementary to the duration of primary transit and the offset between primary and secondary transit (Charbonneau et al. 2005). While technically very challenging, such observations might be more practical than radial velocity observations for host stars that are not amenable to radial velocity observations (e.g., fast rotators, active stars, faint stars, and perhaps low-mass stars whose radiation peaks in the infrared). 


\section{Transit timing variations}

For stars harboring multiple planets, the transit times will not be exactly periodic. By measuring the time of many transits it will be possible to search for additional planets, regardless of whether they are transiting (Miralda-Escudé 2002; Agol et al. 2005; Holman \& Murray 2005; Ford \& Holman 2007). While it is trivial to predict the transit timing variations for a known system, the inverse problem of determining planet masses and orbits from a series of transit times is extremely challenging (Steffen \& Agol 2005; Agol \& Steffen 2007; Veras \& Ford 2008). The amplitude of transit timing variations is particularly large for systems in or near certain mean motion resonances. For some resonances (e.g., 2:1) the amplitude of transit timing variations is large even if the transiting planet follows a circular orbit, but for other resonances (e.g., 3:1) the amplitude is small for nearly circular orbits but large for significant eccentricities. Thus, transit timing variations could be particularly sensitive to eccentricity for transiting planets in certain mean motion resonances.

\section{Implication for Detection \& Characterization of Exoplanets}

\section{Rejecting Transit Search Candidates}

Both planets that transit near pericenter and non-central transits of planets on nearly circular orbits result in a reduced transit duration, so planet searches do not reject such events. However, significantly extended transits only arise due to planets with a correspondingly significant eccentricity. If transit searches were to reject such events (Tingley $\&$ Sackett 2005), then they would decrease their sensitivity for detecting eccentric planets.

\section{Sensitivity of Transiting Planet Searches}

The number of planets detected by transit surveys is a complicated function of survey characteristics and the actual distribution of planets. Simulations can be used to translate the distribution of planet detections into constraints on the actual distribution of planets (Beatty \& Gaudi 2008). Planets that spend less time in transit are harder to detect, both because there is less integrated signal and because better temporal sampling is required to observe the star during transit. This makes it more challenging to detect (or eliminate) planets that transit near pericenter. Fortunately, the reduced planet-star separation increases the probability that an eccentric planet will pass in front of the host star as seen from Earth. The net effect is likely a modest overall increase in the number of detectable transiting planets, but the magnitude of the effect depends on the intrinsic stellar variability and properties of measurement errors (Burke 2008).

\section{Detecting/Rejecting Transits of a Known Planet}

Even for exoplanets discovered by other techniques, detecting or rejecting transits with photometric follow-up observations can be considerably more difficult when allowing for the possibility of eccentric planets. Again, a shorter transit duration reduces the signalto-noise and is more likely to be missed due to poor temporal coverage. When reporting observations that exclude a transit, we encourage observers to specify the shortest transit that would have been detected.

\section{When to look for secondary eclipse}

Even a single transit light curve may provide sufficiently compelling evidence to motivate follow-up observations, particularly for long-period planets. If the putative planet 
were known to follow a circular orbit, then the time of the subsequent transit could be predicted based on the transit duration, modulo modest uncertainties in the transit duration and stellar size (Yee \& Gaudi 2008). Unfortunately, allowing for the possibility of a significant eccentricity will greatly expand the range of potential times for the subsequent transit. Similarly, plans to search for a secondary transit should be mindful of how the uncertainties in orbital eccentricity propagate to increase the uncertainty in time of secondary transit, even once multiple primary transits have been observed. When reporting observations that exclude either a primary or secondary transit, we encourage observers to specify the time window of the relevant observations. A Bayesian approach in which one calculates the distribution of predicted times of future transits could aid in planning follow-up observations and judiciously allocating observing time.

\section{When to confirm small planets with Rossiter Effect}

For terrestrial-mass planet candidates identified via transit searches such as Kepler, it will be extremely challenging to obtain radial velocity confirmation. For planets orbiting stars with favorable properties, it may be possible to confirm the planet via the RossiterMcLaughlin effect (Gaudi \& Winn 2007). Detecting this effect will require large groundbased observatories to make well-timed spectroscopic observations during and around transit. For particularly interesting long-period planets (e.g. near the habitable zone of a G star), the transit duration lasts longer than an observing night from a single observatory. Since the highest precisions are achieved via differential observations, it will be particularly important to time observations (and choose observatories) so as to include the time when the Rossiter-McLaughlin effect is changing the most. If the transit duration has not yet been precisely measured, then such observations should span a time window sufficient to ensure quality measurements both before and after either ingress or egress.

\section{Searching for transiting Trojan planets}

Combining radial velocity and transit observations can be used to search for a Trojan planet companion to a known transiting planet. The time offset between the time of transit and the radial velocity node is sensitive to both the mass of a putative Trojan companion and the eccentricity of the known planet (Ford \& Gaudi 2006; Madhusudhan $\&$ Winn, this volume). Thus, the sensitivity for this method to detect a Trojan planet is set by how well other methods $(\S 2)$ constrain the eccentricity. Conversely, if an offset were observed and a Trojan companion can be excluded as the cause (e.g., by photometric observations spanning more than one libration period), then the offset would be indicative of a non-zero eccentricity.

\section{Impact Parameter}

Since the duration of transit is sensitive to both the impact parameter and the planet's eccentricity, the transit duration alone is not sufficient to measure either. This degeneracy can be broken by measuring the duration of ingress and/or egress with highcadence photometry and/or by measuring the eccentricity via an independent technique $($ see $\S 2)$.

\section{Star $\&$ planet properties estimated from transit light curves}

Traditionally, the stellar properties (e.g., mass, radius) of a field star are inferred by comparing the stellar luminosity, effective temperature, and surface metallicity to stellar models. For favorable targets with accurate parallaxes, this technique can result in random errors of $\simeq 5 \%$ (Takeda et al. 2006). Recently, several groups have attempted to 
determine stellar properties more precisely by incorporating a constraint on the stellar density based on the transit duration. If a planet is known to be on a circular orbit, then the stellar density can be precisely measured via transit photometry alone (e.g., Torres et al. 2008). However, even for systems with several radial velocity observations, the uncertainty in the eccentricity can significantly increase the uncertainty in the stellar density (and other physical properties such as mass and radius). It is important to state explicitly whether an estimate of the stellar density assumes a circular orbit or whether one has accounted for the uncertainty in the eccentricity.

The uncertainty in the radius of a transiting planet is typically limited by the uncertainty in the radius of the host star. Therefore, the possibility of an eccentric orbit reduces measurement precision for the planet radius, density, surface gravity, and structure.

\section{Measuring Eccentricities for Planets in Multiple Planet Systems}

Observations capable of constraining eccentricities from transit light curves (see $\S 2$ ) could be particularly advantageous for studying transiting planets that reside in multiple planet systems. Since the stellar radial velocity is affected by all planets, additional undetected planets may cause radial velocity perturbations that could be misinterpreted as an eccentricity. However, the properties of a given transit light curve are determined by the position and velocity of a single planet (at the epoch of transit). For systems with sufficient radial velocity observations, transit light curves provide an independent check of the eccentricity and could provide early indications suggestive of additional planetary companions.

\section{Acknowledgements}

We appreciate feedback from Sam Quinn, Dimitri Veras, and the Kepler science team. EBF is supported by the University of Florida, and KDC is supported by a University of Florida Alumni Fellowship.

\section{References}

Agol, E., Steffen, J., Sari, R., \& Clarkson, W. 2005, MNRAS, 359, 567

Agol, E. \& Steffen, J. H. 2007, MNRAS, 374, 941

Bakos, G. Á., et al. 2007, ApJ, 670, 826

Barnes, J. W. 2007, PASP, 119, 986

Beatty, T. G. \& Gaudi, B. S. 2008, arXiv, 804, arXiv:0804.1150

Burke, C. J. 2008, ApJ, 679, 1566

Butler, R. P., et al. 2006, ApJ, 646, 505

Carter, J. A., Yee, J. C., Eastman, J., Gaudi, B. S., \& Winn, J. N. 2008, arXiv, 805, arXiv:0805.0238

Charbonneau, D., et al. 2005, ApJ, 626, 523

Christian, D. J., et al. 2008, arXiv, 806, arXiv:0806.1482

Claret, A. 2000, A\&A, 363, 1081

Deming, D., Harrington, J., Laughlin, G., Seager, S., Navarro, S. B., Bowman, W. C., \& Horning, K. 2007, ApJ, 667, L199

Ford, E. B. 2006, ApJ, 642, 505

Ford, E. B. \& Gaudi, B. S. 2006, ApJ, 652, L137

Ford, E. B. \& Holman, M. J. 2007, ApJ, 664, L51

Ford, E. B., Quinn, S. N., \& Veras, D. 2008, ApJ, 678, 1407

Gaudi, B. S. \& Winn, J. N. 2007, ApJ, 655, 550

Gillon, M., Triaud, A. H. M. J., Mayor, M., Queloz, D., Udry, S., \& North, P. 2007, arXiv, 712, arXiv:0712.2073

Holman, M. J. \& Murray, N. W. 2005, Sci, 307, 1288 
Johns-Krull, C. M., et al. 2008, ApJ, 677, 657

Jordan, A. \& Bakos, G. A. 2008, arXiv, 806, arXiv:0806.0630

Joshi, Y. C., et al. 2008, arXiv, 806, arXiv:0806.1478

Laughlin, G., Marcy, G. W., Vogt, S. S., Fischer, D. A., \& Butler, R. P. 2005, ApJ, 629, L121

Mandel, K. \& Agol, E. 2002, ApJ, 580, L171

Maness, H. L., Marcy, G. W., Ford, E. B., Hauschildt, P. H., Shreve, A. T., Basri, G. B., Butler, R. P., \& Vogt, S. S. 2007, PASP, 119, 90

Miralda-Escudé, J. 2002, ApJ, 564, 1019

Shen, Y. \& Turner, E. L. 2008, arXiv, 806, arXiv:0806.0032

Southworth, J. 2008, MNRAS, 386, 1644

Ribas, I., Font-Ribera, A., \& Beaulieu, J.-P. 2008, ApJ, 677, L59

Steffen, J. H. \& Agol, E. 2005, MNRAS, 364, L96

Takeda, G., Ford, E. B., Sills, A., Rasio, F. A., Fischer, D. A., \& Valenti, J. A. 2007, ApJS, 168, 297

Tingley, B. \& Sackett, P. D. 2005, ApJ, 627, 1011

Torres, G., Winn, J. N., \& Holman, M. J. 2008, ApJ, 677, 1324

Winn, J. N., Holman, M. J., \& Roussanova, A. 2007, ApJ, 657, 1098

Yee, J. C. \& Gaudi, B. S. 2008, arXiv, 805, arXiv:0805.1936 

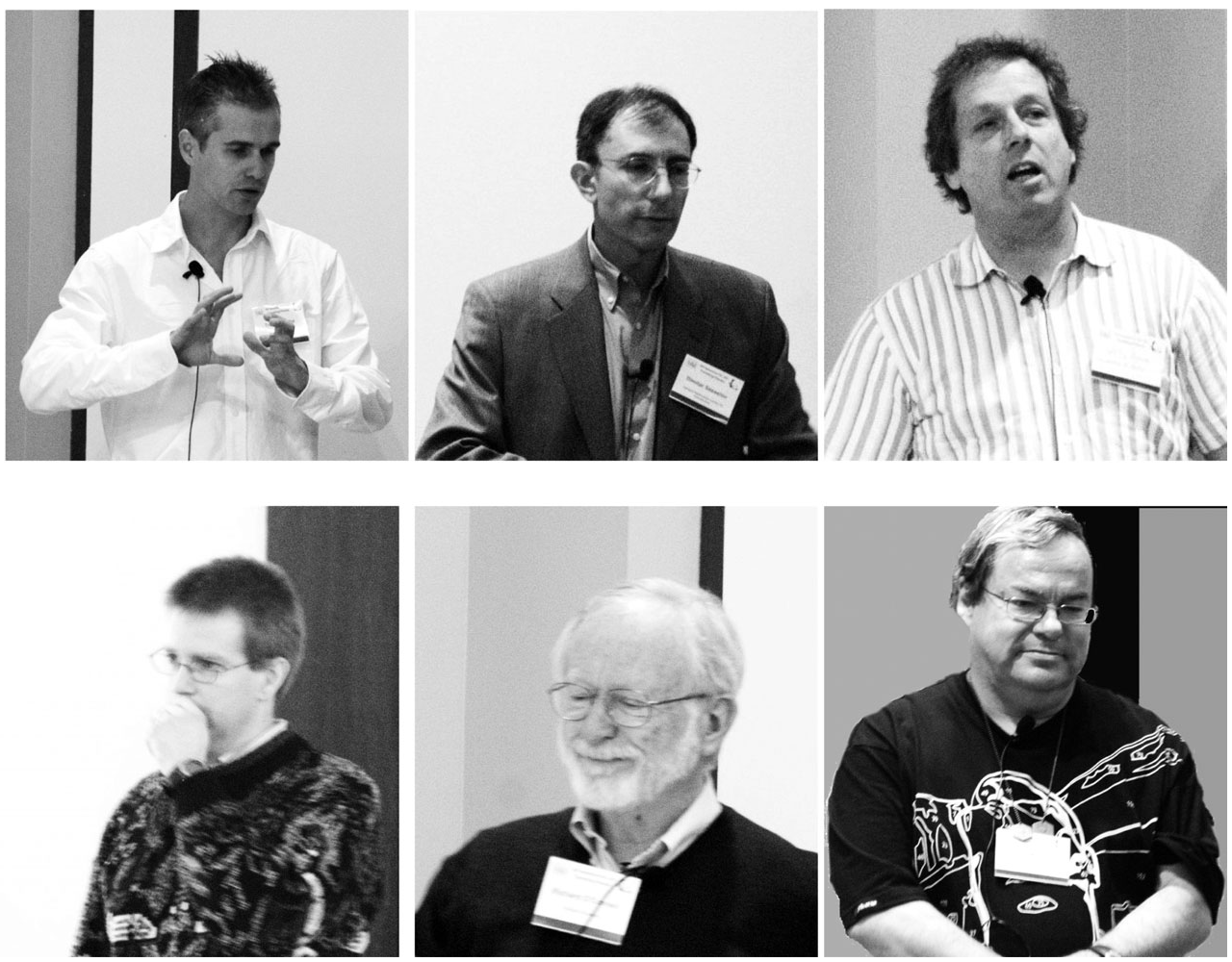

From top left: Tristan Guillot, Dimitar Sasselov, Willy Benz, Adam Showman, Richard O'Connell, Jaymie Matthews

Six speakers invited to the IAU 253 Symposium do not present a contribution to this volume. Recent reviews can be found in:

Guillot 2007: The composition of transiting giant extrasolar planets, Nobel Symposium 135, Stockholm, arXiv:0712.2500

Sasselov, D. D.; Valencia, D.; O'Connell, R. J. 2008: Massive terrestrial planets (superEarths): detailed physics of their interiors, Nobel Symposium 135, Stockholm (Physica Scripta, Volume 130)

Benz, W. et al. 2008: Giant planet population synthesis: comparing theory with observations, Nobel Symposium 135, Stockholm, (Physica Scripta, Volume 130)

Showman, A. P. et al. 2007: Atmospheric Circulation of Hot Jupiters: A Review of Current Understanding, proceedings of the Conference on Extreme Solar Systems held in Santorini, Greece, June 25-29 2007, arXiv:0710.2930

The MOST mission (PI J. Matthews): http://www.astro.ubc.ca/MOST 